\title{
Sufasalazine unveils a contact-independent HSV-TK/ganciclovir gene therapy bystander effect in malignant gliomas
}

\author{
PIERRE A. ROBE, MINH-TUAN NGUYEN-KHAC, FREDERIC LAMBERT, CHANTAL LECHANTEUR, \\ OLIVIER JOLOIS, PATRICIA ERNST-GENGOUX, BERNARD ROGISTER and VINCENT BOURS
}

\author{
Center for Biomedical Integrated Genoproteomics (CBIG) and Center for Cellular \\ and Molecular Neurobiology (CNCM), University of Liège, Belgium
}

Received July 19, 2006; Accepted September 11, 2006

\begin{abstract}
The efficacy of HSV-TK/ganciclovir-based gene therapy on malignant gliomas largely relies on the amplitude of the bystander effect. In these experiments, the antiinflammatory drug Sulfasalazine increased the HSV-TK/ ganciclovir bystander effect in C6, 9L and LN18 cells but not in U87 glioma cells. Using bi-compartmental culture devices and conditioned medium transfer experiments, we showed that in C6, 9L and LN18 cells but not in U87 cells, Sulfasalazine also unveiled a new, contact-independent mechanism of HSV-TK/ganciclovir bystander effect. Upon treatment with ganciclovir, human LN18-TK but not U87-TK cells synthetized and released TNF- $\alpha$ in the culture medium. Sulfasalazine sensitized glioma cells to the toxic effect of TNF- $\alpha$ and enhanced its secretion in LN18-TK cells in response to GCV treatment. The caspase- 8 inhibitor Z-IETD-FMK and a blocking antibody to TNF- $\alpha$ both inhibited the contactindependent bystander effect in LN18 cells. Taken together, these results suggest that TNF- $\alpha$ mediates the contactindependent bystander effect in LN18 cells. The treatment with GCV and/or Sulfasalazine of tumor xenografts consisting of a mix of $98 \%$ C6 and 2\% C6-TK cells shows that Sulfasalazine is also a potent adjunct to the in vivo treatment of gliomas.
\end{abstract}

\section{Introduction}

The efficacy of Herpes virus thymidine kinase/ganciclovir suicide gene therapy largely depends on the ability of transfected cells to kill neighboring untransfected cancer cells, a process called the bystander effect. This effect explains the complete regression of some experimental tumors where only one tenth of the cells are transfected with the suicide gene

Correspondence to: Dr Pierre A. Robe, Department of Human Genetics, University Hospital of Liège, Domaine du Sart Tilman, B35, 4000 Liège, Belgium

E-mail: pierre.robe@ulg.ac.be

Key words: glioma, gene therapy, Sulfasalazine, thymidine kinase, ganciclovir, bystander effect
$(1,2)$, and is believed to arise notably from the diffusion of phosphorylated ganciclovir molecules through gap junctions between transfected and untransfected cancer cells (3-5). Additional mechanisms may also contribute to this bystander effect, such as the phagocytosis of transfected cell debris containing thymidine kinase or the release of yet undefined diffusible death signals $(6,7)$. In vivo, immune mechanisms and vascular occlusion resulting from endothelial cell damage may also occur (8-10). As a wealth of glial tumors display low levels of gap junctional intercellular communication (11), the characterization of the multiple molecular pathways underlying the bystander effect is paramount, and is a prerequisite to the improvement of current gene therapy protocols.

Recent evidence suggests that ligand-independent clustering of a TNF-receptor family death receptor, FAS/CD95, contributes to chemotherapy-induced apoptotic death of cancer cells, and might occur in HSV-tk expressing cells treated with ganciclovir (12). Although one study also suggests that FAS ligand-dependent activation of this same receptor might participate to the bystander effect of some cell types (13), the question of the implication of death receptors in the genesis of the bystander effect remains largely unanswered. Death receptor activation in fact triggers both caspase- 8 dependent apoptosis and an NF-кB-dependent anti-apoptotic cascade in a variety of tumors, including malignant gliomas (14-16). Since the balance between these pro- and anti-apoptotic cascades appears to be altered in glioblastomas (17), we have sought to establish whether Sulfasalazine, a known NF-кB inhibitor (18) and modulator of death receptor function (19), could enhance the efficacy of HSV-TK/ganciclovir gene therapy in gliomas.

\section{Materials and methods}

Cell cultures. C6 cells (ATCC) were kept in DMEM supplemented with $3 \%$ fetal bovine serum (FBS) and penicillin (DEM-C). 9L cells (gift from Dr C. Grignet, Liège, Belgium), LN18 cells (obtained from Dr N. De Tribolet) and U87 cells (ATCC) were grown in RPMI-1640 containing L-glutamine and supplemented with 10\% FBS and penicillin (RPMI-C). Clones LN18-TK, C6-TK and U87-TK were obtained by transfection with a defective murine retrovirus carrying the HSV-tk and the geneticin resistance genes (gift of Dr C. Grignet), selection in G418, and clonal expansion and selected on the basis of their sensitivity to ganciclovir and of their 
growth characteristics similar to those of parental LN18, C6 and U87 cells, respectively $(20,21)$. Clone $9 \mathrm{~L}-\mathrm{TK}$ was a gift from Dr C. Grignet (22). All clones were kept in RPMI-C or DEM-C supplemented with G418.

Cytotoxicity assay. Cytotoxicity assays were performed strictly as described previously $(13,20,21)$. Cells were grown on 96well plates for various periods of time and treated with GCV or TNF- $\alpha$ in the presence or not of sulfaslazine $(0.5 \mathrm{mM})$. MTT assay was then performed. Survival of GCV-treated cells was expressed in percents of the survival of GCV-free cells. Survival of GCV and Sulfasalazine-treated cells was expressed as percentage of the survival of Sulfasalazine-only treated cells. Survival of TNF $\alpha$-treated cells was expressed as percentage of the survival of TNF $\alpha$-free cells. Survival of $\mathrm{TNF} \alpha+$ Sulfasalazine-treated cells was expressed as percentage of the survival of Sulfasalazine-only treated cells.

Assessment of the bystander effect in mixed cultures. The bystander effect was also assessed as described previously $(13,20,21)$. Briefly, $\mathrm{TK}^{+}$and $\mathrm{TK}^{-}$cells were mixed in various proportions and grown in RPMI-C. Treatment conditions were set as described in the results section, media were changed every two days, and the MTT test was used on day 5 to assess total cell survival (23). Survival of GCV-treated cells was expressed as percentage of the survival of GCVfree cells. Survival of GCV + Sulfasalazine-treated cells was expressed as percentage of the survival of Sulfasalazine-only treated cells.

Electric mobility shift assay. Electrical mobility shift assays (EMSA) were performed on nuclear protein extracts as described previously (16) using an oligonucleotide probe identical to the human immunodeficiency virus $\kappa \mathrm{B}$ element (5'-GATCAGGGACTTTCCGCTGGGGACTTTCCAG-3') that was annealed with its complementary strand and endlabeled with [ $\left.{ }^{32} \mathrm{P}\right]$-ATP. Negative (competition) controls were performed with nuclear extracts from HCT-116 cells stimulated with TNF- $\alpha$ for $30 \mathrm{~min}$ and in the presence of an excess of unlabeled probe, whereas positive controls were performed with these same extracts in the presence of an excess of mutant probe (5'-GATCACTCACTTTCCGCTGCTCACTT TCCAG-3').

TNF- $\alpha$ ELISA. LN18-TK or U87-TK cells $\left(10^{6}\right)$ were grown in RPMI medium in the presence or absence of Sulfasalazine $(0.5 \mathrm{mM})$ for $72 \mathrm{~h}$ in $35-\mathrm{mm}$ dishes, and GCV was added at various time-points without replacing the medium. Supernatants were centrifuged to eliminate cell debris and processed with a TNF- $\alpha$ ELISA kit (Peprotech, Rocky Hill, NJ) according to the manufacturer's instructions.

Quantitative RT-PCR. TNF- $\alpha$ mRNA levels were determined with reverse transcription-polymerase chain reaction (RT-PCR) (24) using a TaqMan ${ }^{\circledR}$ (Stratagene, La Jolla, CA) real-time PCR device. Total RNA was extracted from glioma cells using RNEasy columns (Qiagen, Valencia, CA), according to the manufacturer's instructions. Recovered RNA was quantified by spectrometry (Gene Quant, Pharmacia, Piscataway, NJ), and $2 \mu \mathrm{g}$ of RNA was subjected to reverse transcription using the SuperScript First-Strand Synthesis system for RT-PCR (Invitrogen Life Technologies, Paisley, UK). The newly transcribed complementary DNA (cDNA) was amplified by TaqMan quantitative PCR according to the manufacturer's instructions and using SYBR green 1 dye.

Primers were designed to amplify a 227-bp segment on exons 2, 3 and 4 of TNF- $\alpha$ (sense: CCCAGGCAGTCAGATC ATCTTC and antisense: TGGTTATCTCTCAGCTCCACGC) and were standardized with respect to ABL expression.

Assessment of the gap junction intercellular communication (GJIC). FACS analysis of the GJIC was performed as described elsewhere (21). Briefly, cells grown in Petri dishes for $24 \mathrm{~h}$ in the presence or absence of Sulfasalazine were labelled for one hour with either gap-junction diffusible calcein or permanent cytoplasmic dye CMTMR in PBS, rinsed and then mixed at a total density of $2 \times 10^{6}$ cells $/ 6 \mathrm{~cm}$ Petri dish. After another 4-h period in the presence or absence of Sulfasalazine, they were trypsinized, collected in PBS and processed for FACS analysis to evidence the diffusion of calcein to CMTMRstained cells.

Medium transfer experiments. $\mathrm{TK}^{+}$cells were grown for 48-96 h at confluence in 5-ml/9-cm Petri dishes in the presence or absence of GCV $(10 \mu \mathrm{M})$ and/or Sulfasalazine (0.5 mM). Media were then collected, centrifuged at 5000 RPM. Supernatants were filtered on Millex ${ }^{\circledR}$ filters (Millipore, Brussels, Belgium) and transferred for $24 \mathrm{~h}$ on $104 \mathrm{TK}^{-}$cells grown in 96-well plates. Cell survival was then assessed with the MTT test. Controls were set as follows: conditioned medium from untreated $\mathrm{TK}^{+}$cells for $\mathrm{GCV}$-treated $\mathrm{TK}^{+}$cells, and conditioned medium from Sulfasalazine-treated cells for $\mathrm{GCV}+$ Sulfasalazine treated $\mathrm{TK}^{+}$cells. Survival was expressed as percentage of the survival in these control conditions. In some experiments, the caspase-8 inhibitor Z-IETD-FMK (30 $\mu \mathrm{M})$ or a blocking antibody to TNF- $\alpha(25-50 \mathrm{ng} / \mathrm{ml})$ were added to the conditioned media immediately prior to its transfer onto $\mathrm{TK}^{-}$cells.

Bi-compartmental cultures. For each cell type, 20,000 TK- cells were seeded in a 24-well plate and allowed to attach for $1 \mathrm{~h}$. Laminin-coated $(10 \mu \mathrm{g} / \mathrm{ml})$ Millicel ${ }^{\circledR}$ culture inserts then were laid in these wells, and $200,000 \mathrm{TK}^{+}$cells were seeded on these inserts. This system allowed $\mathrm{TK}^{+}$and $\mathrm{TK}^{-}$cells to grow in physically distinct compartments but in the same medium that could be supplemented with $10 \mu \mathrm{M} \mathrm{GCV}$ and/or $0.5 \mathrm{mM}$ Sulfasalazine. After $48-72 \mathrm{~h}, \mathrm{TK}^{-}$cells were rinsed in PBS, trypsinized, and counted in a Thomas cytometer. The experiments were run three times in duplicate.

Western blot experiments. Western blots were performed as described elsewhere (16). Briefly, whole cells protein extracts were separated on $12.5 \%$ Tris- $\mathrm{HCl}$ polyamide gels (Bio-Rad, Nazareth, Belgium), transferred on a PVDF membrane (Amersham) and probed with monoclonal antibodies to FAS-L (B\&D, NOK-1), FAS (Santa-Cruz) and/or Actin (Santa Cruz). A secondary, HRP-coupled, antimouse antibody (Santa Cruz) was then used for the secondary step and reveled using a chemiluminescent substrate (Amersham). 
Table I. Effect of Sulfasalazine on the toxicity of GCV in glioma cells.

\begin{tabular}{lllc}
\hline Cell type & \multicolumn{1}{c}{ Treatment } & $\begin{array}{c}\text { Survival } \\
(\%)\end{array}$ & $\begin{array}{c}\text { Paired Student's } \\
\text { t-test }\end{array}$ \\
\hline LN18-TK & CTRL & $54 \pm 6.32$ & - \\
& Sulfasalazine & $16.7 \pm 7.8$ & $<0.05$ \\
C6-TK & CTRL & $81.9 \pm 5.4$ & - \\
& Sulfasalazine & $70.6 \pm 8.6$ & $<0.05$ \\
9L-TK & CTRL & $57.3 \pm 3.5$ & - \\
& Sulfasalazine & $46.2 \pm 1.6$ & $<0.05$ \\
U87-TK & CTRL & $86.4 \pm 4.1$ & - \\
& Sulfasalazine & $85.5 \pm 12.7$ & $\mathrm{NS}$ \\
\hline
\end{tabular}

In vivo experiments. Fifty thousand C6 mixed with $2 \%$ of C6TK cells were stereotactically implanted in the striatum of 5week old Balb-c nude mice and allowed to grow for 3 days. Mice were then treated for one week with daily intraperitoneal injections of saline (control group), ganciclovir $(100 \mathrm{mg} / \mathrm{kg})$, Sulfasalazine $(15 \mathrm{mg} / \mathrm{kg})$ or Sulfasalazine + ganciclovir. Forty mice were included in the protocol (i.e., 10 animals per group). Animals were sacrificed on day 30 and the brains immediately fixed in formalin. The brains were cut in consecutive 1-mm thick slices using an adult mice brain coronal slicer (WPI, Sarasota, FL), stained with cresyl violet and photographed using a Zeiss Axiocam camera mounted on a Zeiss stereoscopic magnifying loupes system. The tumor contours and surface measurements were performed using the NIH ImageJ (version 1.33u) software. Volumes were obtained by multiplication of the individual tumor surfaces by their constant 1-mm thickness. Statistical analyses were performed using Statview Version 5.0 software (SAS institute).

\section{Results}

Effects of Sulfasalazine on the toxicity of GCV in glioma cells. Sulfasalazine significantly increased the sensitivity of TK-expressing LN18, C6 and 9L glioma cells to GCV. In LN18-TK cells and after $72 \mathrm{~h}$ of treatment with GCV, survival in control conditions was $54 \pm 6.34 \%$ but only $16.7 \pm 7.8 \%$ in the presence of Sulfasalazine. The survival of C6-TK cells after $48 \mathrm{~h}$ of treatment with GCV alone was $81.9 \pm 5.4 \%$ of that in control wells. but decreased to $70.6 \pm 8.6 \%$ with Sulfasalazine co-treatment. In 9L-TK treated for $72 \mathrm{~h}$ with GCV, survival decreased from $57.3 \pm 3.5 \%$ in control conditions to $46.2 \pm 1.6 \%$ in the presence of Sulfasalazine $(n=3, p<0.05$, paired Student's t-test for all cell lines, Table I).

The survival following GCV treatment of U87-TK cells for $72 \mathrm{~h}$ was, however, unaffected by Sulfasalazine treatment $(86.4 \pm 4.1 \%$ in control conditions versus $85.5 \pm 12.7 \%$ in the presence of Sulfasalazine).

Effects of Sulfasalazine on the bystander effect of cells grown in contact. Sulfasalazine slightly but significantly increased

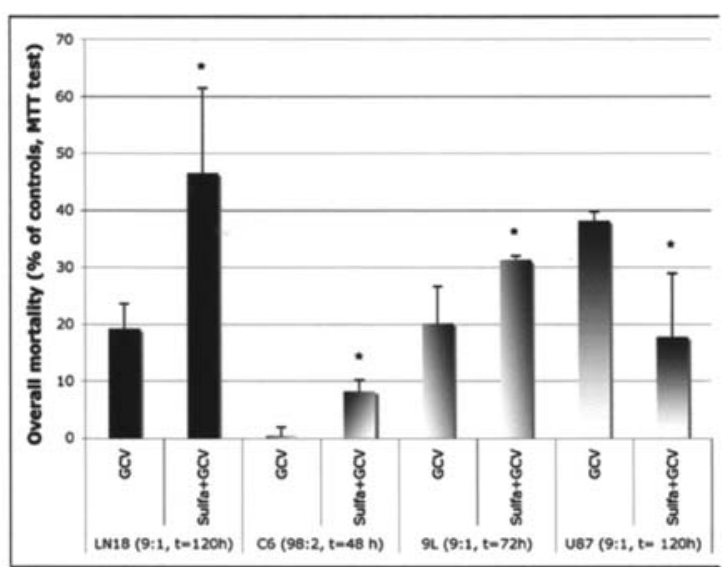

Figure 1. Effect of Sulfasalazine on the bystander effect of glioma cells grown in contact. Wild-type LN18, C6, 9L or U87 cells were mixed with their respective TK-expressing counterparts and grown in the presence or absence of ganciclovir and/or sulfasalazine $(10 \mu \mathrm{M})$. After a period of time indicated in the chart, total cell survival was assessed in each condition with the MTT test. The overall mortality represents the difference of survival between GCV-treated and GCV-free wells, expressed as percentage ( $\left.{ }^{*} \mathrm{p}<0.05\right)$.

the HSV-TK/GCV bystander effect in LN18, C6 and 9L cells. In cultures of LN18 and 10\% LN18-TK cells (9:1 cultures) grown for 5 days in the presence of $\mathrm{GCV}(10 \mu \mathrm{M})$, the overall mortality augmented significantly from $19.1 \pm 4.4 \%$ to $45.62 \pm 15 \%$ after treatment with Sulfasalazine $(0.5 \mathrm{mM})$. In 98:2 cultures of C6 and C6-TK cells grown for $48 \mathrm{~h}$ in the presence of GCV $(10 \mu \mathrm{M})$, mortality increased from $0.4 \pm 1.5 \%$ in control to $8.3 \pm 2 \%$ in the presence of Sulfasalazine. In 9L cells cultured for 3 days with $10 \%$ of 9L-TK cells and treated with $10 \mu \mathrm{M} \mathrm{GCV}$, the overall mortality increased from $20.1 \pm 6.5 \%$ to $31.2 \pm 0.8 \%$ in the presence of $0.5 \mathrm{mM}$ Sulfasalazine ( $n=3, p<0.05$, Student's t-test for all cell lines).

However, in 9:1 cultures of U87 and U87-TK cells grown for 5 days, the overall mortality in control conditions was $38.1 \pm 1.6 \%$ but decreased to $17.7 \pm 11.2 \%$ in the presence of Sulfasalazine (Fig. 1, p $<0.05$, paired Student's t-test).

Sulfasalazine does not alter gap junction intercellular communication (GJIC) in glioma cells. Using a FACS analysis of the intercellular transfer of calcein dye in C6, 9L, U87 and LN18 cells, we could not evidence any effect of Sulfasalazine on the intercellular communication (Fig. 2A and data not shown).

Effects of GCV and Sulfasalazine on $N F-\kappa B$ nuclear binding activity in glioma cells. We found that in C6-TK, 9L-TK and LN18-TK cells the constitutive NF- $\mathrm{KB}$ nuclear activity was increased after ganciclovir treatment but returned to baseline following low-dose $(0.5 \mathrm{mM})$ Sulfasalazine treatment. In U87-TK cells, we did not observe any GCV-related activation of NF- $\mathrm{B}$, nor did we observe any major inhibition of the baseline NF- $\mathrm{KB}$ activity (Fig. 2B and data not shown).

Sulfasalazine treatment can unveil a contact-independent bystander effect in gliomas. $\mathrm{TK}^{+}$and $\mathrm{TK}^{-}$glioma cells were grown in bi-compartmental culture devices where they were physically separated by a porous membrane. The treatment 
A
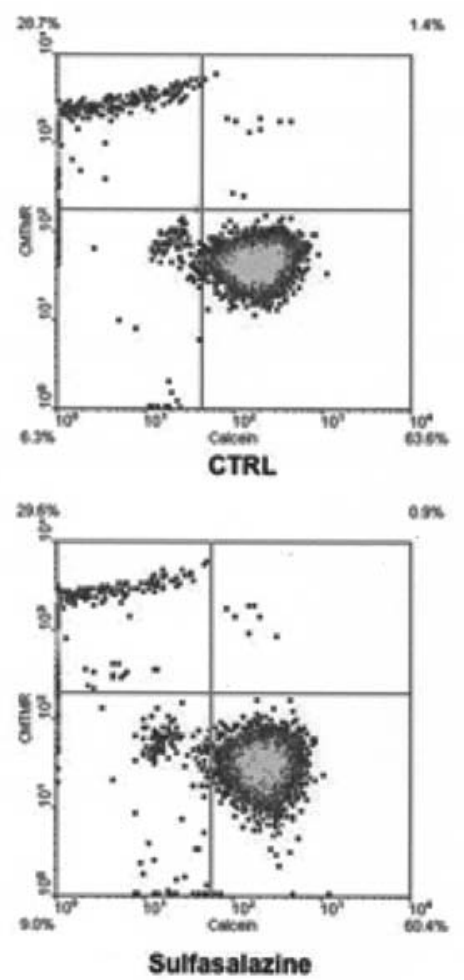

Figure 2. Effect of Sulfasalazine on the GJIC and NF- $\mathrm{\kappa B}$ binding activity in glioma cells. A, CMTMR and Calcein-loaded LN18 cells were mixed and cultured for $4 \mathrm{~h}$ with or without Sulfasalazine. FACS analysis was then performed allowing the simultaneous measurement of CMTMR and Calcein fluorescence. The proportions of CMTMR loaded cells in which Calcein has diffused (right upper quadrants) is a measure of the GJIC. B, NF-кB binding activity in the nuclei of LN18-TK and U87-TK cells following treatment with ganciclovir and Sulfasalazine for $24 \mathrm{~h}(<>$, specific NF- $\mathrm{BB}$ binding activity).

of such co-cultures with GCV did not alter the survival of LN18, C6, 9L or U87 cells, meaning that the bystander effect in these glioma cells is contact-dependent. Co-treatment with Sulfasalazine and GCV, however, unveiled a bystander effect in LN18, C6 and 9L cells but not in U87 cells. In such cocultures, LN18 cell survival decreased from $99.4 \pm 24.5 \%$ in GCV-treated wells to $51.2 \pm 10.6 \%$ in GCV + Sulfasalazinetreated wells after $72 \mathrm{~h}$. The survival of C6 cells grown for $48 \mathrm{~h}$ in such bi-compartmental cultures decreased from $106.1 \pm 15.4 \%$ in $\mathrm{GCV}$ wells to $62 \pm 12.7 \%$ in the presence of GCV and Sulfasalazine. In 9L/9L-TK co-cultures, 9L cell survival similarly decreased from $104.8 \pm 4.6 \%$ to $61.1 \pm 13.9 \%$ (for each cell type, $\mathrm{n}=3, \mathrm{p}<0.05$, Student's t-test). In U87/ U87-TK co-cultures, however, survival of U87 cells after $72 \mathrm{~h}$ of treatment with GCV was $97.9 \pm 4.3 \%$ in control wells versus $94.9 \pm 10.3 \%$ in the presence of Sulfasalazine (NS, Student's t-test, Fig. 3A).

Sulfasalazine did not alter the toxicity of GCV on wildtype (i.e., TK-) C6, LN18, 9L or U87 cells (data not shown), suggesting that the observed loss of survival in C6, LN18 and $9 \mathrm{~L}$ cells grown in bi-compartmental cultures and in the presence of Sulfasalazine and GCV was indeed dependent on the presence of their TK-expressing counterparts in the
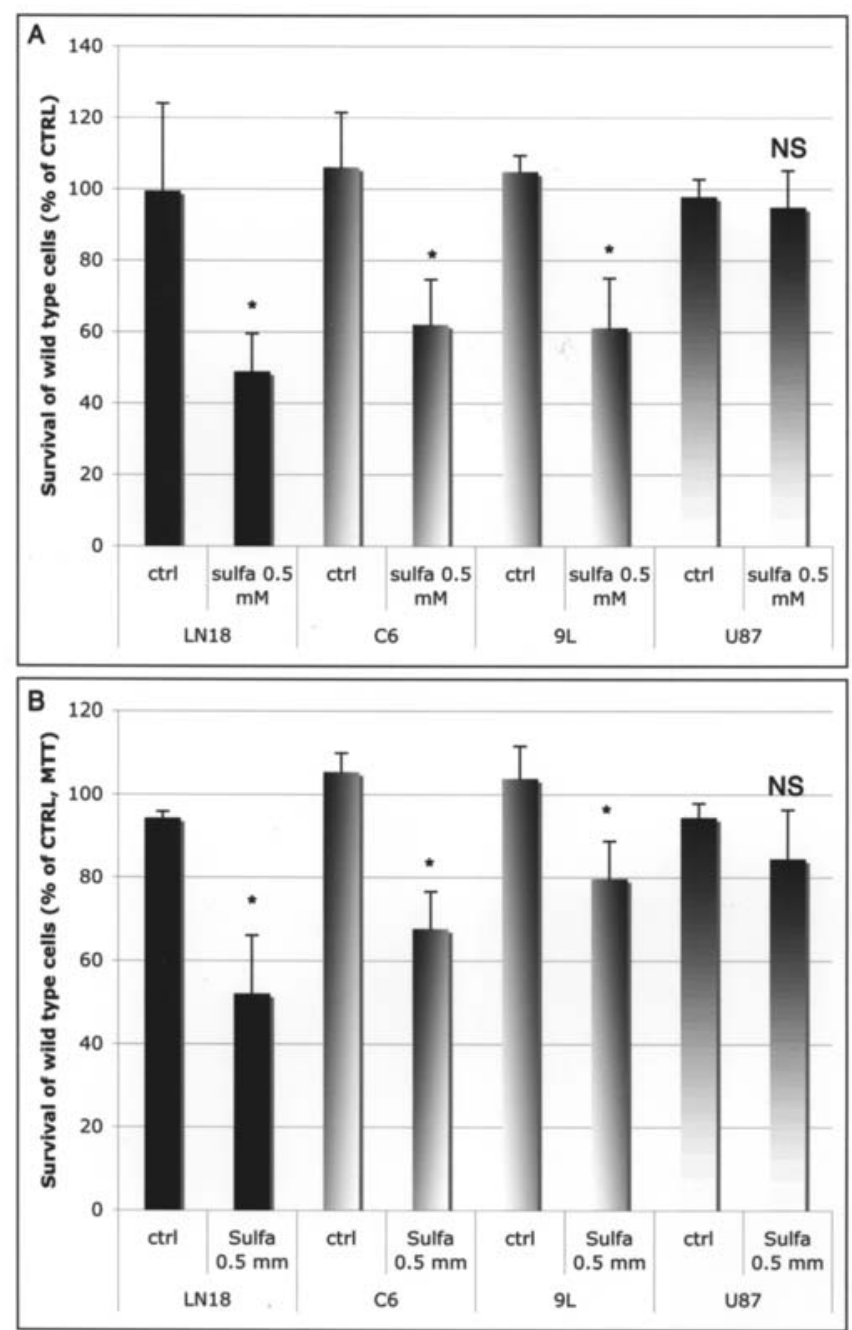

Figure 3. Effect of sulfasalazine on the contact-independent bystander effect of glioma cells. A, Survival of wild-type LN18, C6, 9L and U87 cells grown in bi-compartmental devices where the second compartment contained their respective TK-expressing counterparts. Survival was obtained by the trypanblue exclusion method and is expressed as a percentage with respect to similar cultures grown without ganciclovir, both with and without the adjunction of Sulfasalazine in the culture medium $\left({ }^{*} \mathrm{p}<0.05\right)$. B, MTT-assessed survival of wild-type LN18, C6, 9L and U87 cells grown for $24 \mathrm{~h}$ in medium conditioned in their respective TK-expressing counterparts in the presence or not of ganciclovir and Sulfasalazine $\left({ }^{*} \mathrm{p}<0.05\right)$.

second culture compartment. To test this hypothesis further, conditioned medium of LN18-TK, C6-TK, 9L-TK and U87-TK cells treated or not with Sulfasalazine and/or GCV was added in cultures of wild-type LN18, C6, 9L or U87 cells. Conditioned medium of LN18-TK cells treated with GCV for $72 \mathrm{~h}$ hardly reduced the survival of LN18 cells onto which it was transferred for another $24 \mathrm{~h}(94.2 \pm 1.6 \%$ of controls). Medium conditioned by GCV+Sulfasalazine treated LN18-TK cells, however, strongly decreased the survival of LN18 cells to $52.1 \pm 14 \%$ after such transfer. Conditioned medium of C6-TK cells treated for $48 \mathrm{~h}$ with GCV did not reduce the survival of wild-type C6 cells $(105.3 \pm 4.5 \%)$, whereas the survival of C6 cells was reduced to $67.6 \pm 8.9 \%$ when the conditioned medium was obtained from Sulfasalazine and GCV-treated C6-TK cells. In similar experiments with 9L cells, the $24-\mathrm{h}$ survival decreased from $103.8 \pm 7.8 \%$ in GCV- 

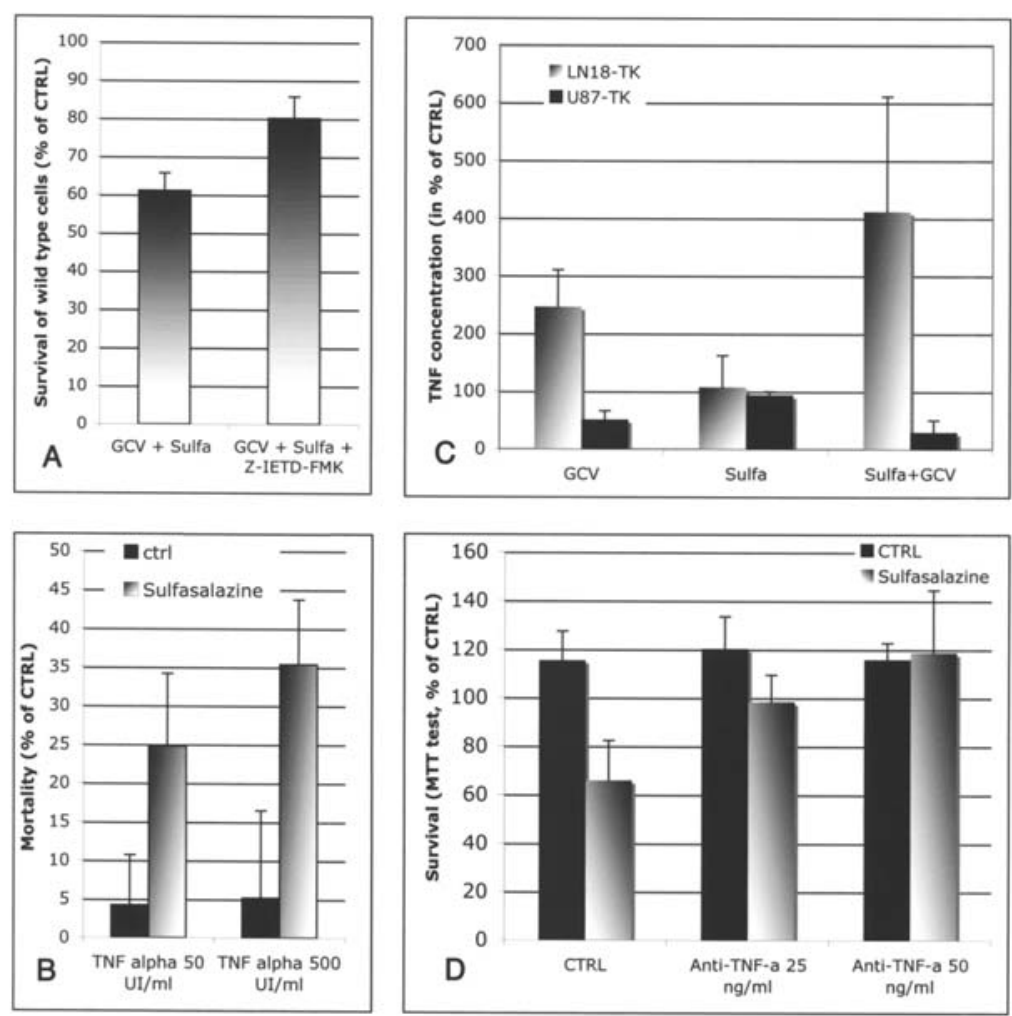

Figure 4. Role of TNF- $\alpha$ in the contact-independent bystander effect of LN18 cells. A, Effect of the caspase- 8 inhibitor on the Sulfasalazine-induced contactindependent bystander effect of LN18 cells grown in medium conditioned in LN18-TK cells. Experiments were performed as in Fig. 3B. B, Effect of Sulfasalazine on the toxicity of TNF- $\alpha$ on LN18 cells. Survival was measured by an MTT test. The mortality represents the difference in percentage between the survival in the presence or absence of TNF- $\alpha$. C, Secretion of TNF- $\alpha$ in the culture medium of LN18-TK cells and U87-TK cells after ttreatment with GCV and/or Sulfasalazine fo $96 \mathrm{~h}$. TNF- $\alpha$ concentrations were measured by ELISA and are expressed relative to the concentration of TNF- $\alpha$ measured for each cell type in the absence of any treatment. D, Effect of a blocking antibody to TNF- $\alpha$ on the Sulfasalazine-unveiled contact-independent bystander effect of LN18 cells grown in medium conditioned in LN18-TK cells. Experiments were performed as in Fig. 3B.

only conditioned medium to $79.7 \pm 9 \%$ in Sulfasalazine + GCV-conditionned medium ( $\mathrm{n}=3, \mathrm{p}<0.05$, Student's t-test). On the contrary, the survival of U87 cells remained unchanged when grown in medium conditioned by GCV or GCV + Sulfasalzine treated U87-TK cells (Fig. 3B).

Caspase-8 inhibitor Z-IETD-FMK inhibits the bystander effect in glioma cells. The caspase-8 inhibitor ZIETD-FMK $(20 \mu \mathrm{M})$ significantly reduced the bystander effect in cocultures of C6 and C6-TK cells. The overall survival of C6 cells cultured for 5 days with $10 \%$ of C6-TK cells and treated with GCV increased from $23.4 \pm 5.7 \%$ to $44.8 \pm 8.8 \%$ in the presence of Z-IETD-FMK ( $\mathrm{n}=3, \mathrm{p}<0.05$, Student's ttest). Used as a control, the cathepsin inhibitor Z-FA-FMK $(20 \mu \mathrm{M})$ did not alter this bystander effect significantly. A similar inhibition of the bystander effect by Z-IETD-FMK was observed in LN18 cells cultured with $50 \%$ of LN-TK cells and treated with GCV. Under these conditions, the overall cell survival increased from $36.8 \pm 11.7 \%$ to $71.6 \pm 23.8 \%(n=3, p<0.05$, Student's t-test $)$.

Addition of the caspase-8 inhibitor Z-IETD-FMK $(30 \mu \mathrm{M})$ also significantly inhibited the toxicity of the conditioned medium of Sulfasalazine and GCV-treated LN18-TK cells on LN18 cells. The 24-h survival of LN18 cells grown in conditioned medium from LN18-TK cells treated with GCV and Sulfasalazine increased from $61.1 \pm 4.6 \%$ to $80.1 \pm 5.6 \%$ when Z-IETD-FMK was added to the conditioned medium immediately before its transfer to LN18 cells $(n=3, p<0.05$, Student's t-test, Fig. 4A).

Sulfasalazine enhances the toxicity of TNF- $\alpha$ on LN18 and U87 cells but not that of soluble FAS-L. Sulfasalazine $(0.5 \mathrm{mM})$ significantly increased the toxicity of $\mathrm{TNF}-\alpha$ on both U87 and LN18 human glioma cells as measured by the MTT test. The treatment of LN18 cells with TNF- $\alpha$ (50 or $500 \mathrm{UI} / \mathrm{ml}$ ) for $24 \mathrm{~h}$ barely reduced the survival of these cells (mortality: $4.2 \pm 6.5$ and $5.17 \pm 11.2 \%$ respectively). In the presence of Sulfasalazine, however, the TNF-induced cell mortality reached $24.7 \pm 9.4$ and $35.3 \pm 8.4 \%$ respectively. Likewise, the treatment of U87 cells with Sulfasalazine increased the toxicity of TNF- $\alpha(100$ or $1000 \mathrm{UI} / \mathrm{ml})$ from $4.1 \pm 4.7$ and $6.7 \pm 2.67 \%$ to $26.6 \pm 7.6$ and $33.1 \pm 17 \%$ respectively $(n=3$, $\mathrm{p}<0.05$, Student's t-test, Fig. 4B and data not shown).

Sulfasalazine $(0.5 \mathrm{mM})$ also significantly increased the cytotoxic action of the FAS activating antibody APO1-3 on LN18 cells. After $24 \mathrm{~h}$, a 1:50 (v:v) dilution of this antibody reduced the survival of LN18 cells by only $7.6 \pm 13.5 \%$. In the presence of Sulfasalazine, this antibody significantly reduced the survival of LN18 cells by $65.1 \pm 10.7 \%(n=3$, $\mathrm{p}<0.05$, Student's t-test). Sulfasalazine significantly protected LN18 cells against soluble FAS-L as the survival of LN18 cells treated with $50 \mathrm{ng} / \mathrm{ml} \mathrm{sFAS-L}$ increased from $54.9 \pm 12.9$ to $89.9 \pm 15.4 \%$ in the presence of Sulfasalazine $(n=3, p<0.05$, Student's t-test). 

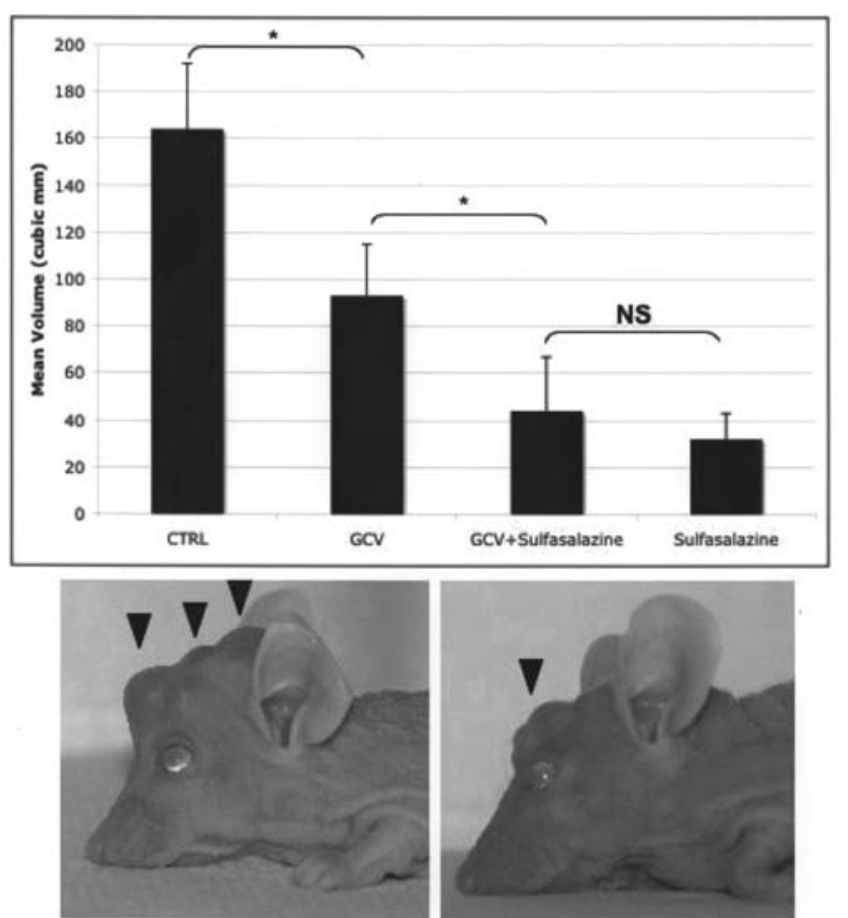

CTRL
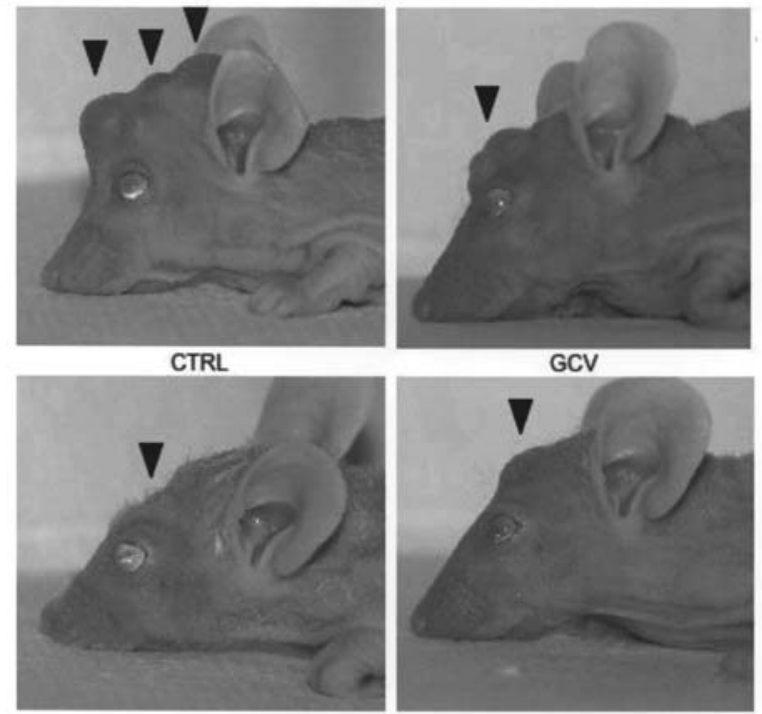

GCV+SULFASALAZINE

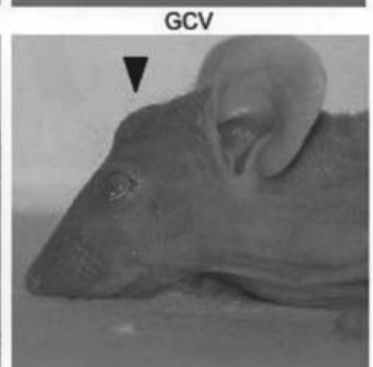

SULFASALAZINE

Figure 5. Effect of Sulfasalazine and GCV on the in vivo growth of chimaeric C6/C6-TK tumors. Fifty thousand C6 cells mixed with $2 \%$ C6-TK cells were injected in the striatum of nude mice (day 0 ). The animals were treated for 1 week with a normal saline solution, GCV alone, Sulfasalazine alone or ganciclovir + Sulfasalazine between days 3 and 10 and allowed to grow for 30 days. The mean volume of tumors in each treatment condition is shown in the graph. Pictures of animals representative of each treatment group are shown at the lower end of the figure. Note the size of the transcranial, subcutaneous growth of tumors in the CTRL and GCV alone conditions with respect to that of Sulfasalazine or Sulfasalazine + GCV treated mice (arrowheads)

GCV treatment increases the secretion of TNF- $\alpha$ in TKexpressing LN18 glioma cells but not in U87-TK cells. Both LN18-TK and U87-TK cells were found to secrete TNF- $\alpha$ in their culture medium. Treatment of LN18-TK cells with GCV $(10 \mu \mathrm{M})$ in serum-free medium more than doubled the secretion of TNF- $\alpha$ within $96 \mathrm{~h}$, and Sulfasalazine co-treatment even increased this phenomenon ( $n=3, p<0.05$, ANOVA). On the contrary, GCV treatment significantly inhibited by half the secretion of TNF- $\alpha$ in U87-TK conditioned media, and this effect was enhanced by Sulfasalazine co-treatment $(n=3$, $\mathrm{p}<0.01$, ANOVA, Fig. 4C).

Quantitative RT-PCR also reproducibly demonstrated a moderate increase of TNF- $\alpha$ mRNA in LN18 cells after $24 \mathrm{~h}$ of treatment with GCV. TNF- $\alpha$ mRNA content, however, remained unchanged in U87 upon such treatment (data not shown).

A blocking antibody to TNF- $\alpha$ inhibits both the contactdependent and contact-independent bystander effect in LN18 human glioma cells. The addition of increasing concentrations of a blocking antibody to TNF- $\alpha$ significantly and dosedependently inhibited the bystander effect in LN18 cells grown in contact with LN18-TK cells at the ratio of 8:2. Indeed, the addition of $40 \mathrm{ng} / \mathrm{ml}$ of blocking antibody to the culture medium increased the overall survival of such cultures after 5 days from $47.3 \pm 6.9$ to $67.8 \pm 12.3 \%$ ( $p<0.05$, ANOVA with Tukey-Kramer Multiple comparison test). Similarly, the addition of this antibody to Sulfasalazine + GCV-treated LN18-TK conditioned medium prior to its transfer onto LN18 cells dose-dependently and significantly inhibited the contact-independent bystander effect (for details, see Fig. 4D; $\mathrm{n}=3, \mathrm{p}<0.05$, ANOVA).

In vivo effect of Sulfasalazine on the growth of C6 tumors containing 2\% C6-TK cells. Fifty thousand C6 and C6-TK cells at the ratio of 98:2 were stereotactically implanted in the striatum of nude mice. After 3 days, the mice were given daily injections of a normal saline solution, ganciclovir (GCV), Sulfasalazine or a combination of these drugs for 1 week and sacrificed after another 3 weeks. The resulting tumor size was significantly reduced in all treatment groups with respect to controls. The tumor size was further reduced in Sulfasalazinetreated and sulfasalzine+GCV-treated animals with respect to GCV-treatment alone. There was, however, no significant difference in the mean tumor size of Sulfasalazine alone and sulfasalzine + GCV treatment groups $(n=10$ in each group, p $<0.05$, ANOVA, Fig. 5).

\section{Discussion}

In this work, we studied the effect of the NF- $\mathrm{KB}$ inhibitor Sulfasalazine on the efficacy and bystander effect of HSV-TK/ ganciclovir gene therapy for malignant gliomas. The inhibition of NF-kB has been shown to potentiate the effect of a variety of cytotoxic treatments including radiation therapy (25-27), chemotherapy (28) and more recently HSV-TK/ ganciclovir gene therapy in glioblastoma (29). We found that GCV treatment activated NF- $\mathrm{KB}$ in $\mathrm{C} 6,9 \mathrm{~L}$ and $\mathrm{LN}-18$ TKexpressing cells but not in U87-TK cells, and that Sulfasalazine both suppressed this activation and sensitized C6, 9L and LN-18 TK-expressing cells to GCV toxicity. Moriuchi et al recently showed that concomitant $\mathrm{I}-\mathrm{kB}$ gene therapy also increased the action of HSV-TK/GCV gene therapy in U87 cells, which we failed to observe with Sulfasalazine treatment. This difference may originate from a less complete inhibition of NF- $\mathrm{KB}$ binding activity in our experiments at the dose of Sulfasalazine used for these cells, or by the existence of NF-кB independent actions of Sulfasalazine on apoptosis in glioma cells (19).

We also found that Sulfasalazine treatment augmented the bystander effect in $\mathrm{C} 6,9 \mathrm{~L}$ and $\mathrm{LN}-18$ cells but not in U87 cells. As this increase did not correlate with an enhanced GJIC between glioma cells in response to Sulfasalazine treatment, we sought to clarify whether soluble factors could account for it. Both bi-compartmental cultures and conditioned medium transfer experiments showed that such a mechanism indeed developed following treatment with Sulfasalazine in 
two rat (C6 and 9L) and one human (LN18) glioma cell lines. This finding is to the best of our knowledge the first report of a medium-diffusible bystander effect in glioma cells using the same strains of $\mathrm{TK}^{-}$and $\mathrm{TK}^{+}$cells. We had, however, observed a similar mechanism in DHD-K12 colon cancer cells (7), and conditioned medium of TFG10.2-TK cells treated with GCV was shown to be toxic to C6 cells (6). Again, U87 cells did not elicit any contact-independent bystander effect.

We then assessed the role of caspase- 8 inhibition on our models of bystander effect, since death receptors and their ligands can participate in HSV-TK/ganciclovir induced cell death and enhance the efficacy of this suicide gene therapy $(12,13,30,31)$. Treatment with the caspase- 8 inhibitor ZIETD-FMK indeed inhibited the contact-dependent bystander effect in C6 and LN18, as well as the contact-independent bystander effect in LN18 cells.

As members of the death receptor superfamily can induce apoptosis through a caspase- 8 dependent pathway, we investigated whether the cytotoxic activity of two secreted activators of these receptors, TNF- $\alpha$ and soluble FAS-L (sFAS-L), was increased after Sulfasalazine treatment. Indeed, this drug sensitized glioma cells to TNF- $\alpha$, an effect that is likely due to its anti-NF-кB properties (17). The toxicity of sFAS-L on the contrary was decreased following treatment with Sulfasalazine, an effect that has previously been reported and is likely NF- $\mathrm{BB}$ independent (19). TNF- $\alpha$ mRNA transcription and protein secretion were also augmented in LN18-TK cells in response to GCV treatment, but decreased in U87-TK cells that lacked any contact-independent bystander effect. A blocking antibody to TNF- $\alpha$ dosedependently inhibited the contact-independent bystander effect in LN18 cells. Taken together, these data on LN18 and U87 cells demonstrate that TNF- $\alpha$ can mediate a contactindependent bystander effect in HSV-TK/GCV gene therapy in the presence of Sulfasalazine. While the transcription factor NF- $\mathrm{KB}$ is a potential regulator of TNF- $\alpha$ synthesis (32), the opposite effect of GCV treatment on the secretion of TNF- $\alpha$ in LN18-TK and U87-TK is unlikely due their differential $\mathrm{NF}-\kappa \mathrm{B}$ response to this drug. Indeed, Sulfasalazine treatment abolished the GCV-induced activation of NF-кB in LN18-TK cells but further increased the secretion of TNF- $\alpha$ by these cells. The precise mechanisms that underlie the difference of behaviour of LN18 and U87 cells were not studied in the present work.

The generation of a contact-independent bystander effect has a theoretical interest in the treatment of gliomas with gene therapy. Indeed, the GJIC of these tumors is highly variable and often decreased (11), and the physical contact between glioma cells is hampered by their tendency to migrate at a distance from the tumoral epicenter $(33,34)$. As our attempts at growing human LN18 cells in the brains of nude mice failed, and since Sulfasalazine does not enhance the bystander effect in human U87 cells, we assessed the in vivo benefit of Sulfasalazine and HSV-TK/GCV gene therapy on C6 cell xenografts. GCV treatment alone significantly decreased the growth of chimeric tumors consisting of $98 \%$ of C6 cells and $2 \%$ of C6-TK cells. The addition of Sulfasalazine further and significantly reduced the growth of such tumors with respect to GCV treatment alone, an effect that was, however, not different from the effect of Sulfasalazine alone. We have demonstrated in the past that Sulfasalazine by itself is a potent inhibitor of glioma growth (16), and this drug is currently being tested in a phase I/II clinical protocol as a treatment for recurrent gliomas in humans (35). Different models of tumors, using diffusely infiltrative tumors that are less sensitive to the sole action of Sulfasalazine may thus be needed to truly confirm the enhancement of the bystander effect by this drug in vivo, but this is beyond the scope of the present study. Our in vivo results nonetheless confirm the absence of a drawback and the potential benefit of adding Sulfasalazine to HSV-TK/ GCV gene therapy in gliomas.

\section{Acknowledgements}

PAR is a Research Associate of the FNRS of Belgium. This work was supported by grants from the FNRS of Belgium, the Fondation Léon Frédéricq, the Centre Anti-Cancéreux près l'Université de Liège, the Belgian Federation against Cancer and the FIRS Fund of the University Hospital of Liège.

\section{References}

1. Freeman SM, Abboud CN, Whartenby KA, Packman CH, Koeplin DS, Moolten FL and Abraham GN: The 'bystander effect': tumor regression when a fraction of the tumor mass is genetically modified. Cancer Res 53: 5274-5283, 1993.

2. Puumalainen AM, Vapalahti M, Agrawal RS, Kossila M, Laukkanen J, Lehtolainen P, Viita H, Paljarvi L, Vanninen R and Yla-Herttuala S: Beta-galactosidase gene transfer to human malignant glioma in vivo using replication-deficient retroviruses and adenoviruses. Hum Gene Ther 9: 1769-1774, 1998.

3. Bi WL, Parysek LM, Warnik R and Stambrook PJ: In vitro evidence that metabolic cooperation is responsible for the bystander effect observed with HSV tk retroviral gene therapy. Human Gene Ther 4: 725-731, 1993.

4. Ishii-Morita H, Agbaria R, Mullen CA, Hirano H, Koeplin DA, Ram Z, Oldfield EH, Johns DG and Blaese RM: Mechanism of 'bystander effect' killing in the herpes simplex thymidine kinase gene therapy model of cancer treatment. Gene Ther 4: 244-251, 1997.

5. Dilber MS, Abedi MR, Christensson B, Bjorkstrand B, Kidder GM, Naus CC, Gahrton G and Smith CI: Gap junctions promote the bystander effect of herpes simplex virus thymidine kinase in vivo. Cancer Res 57: 1523-1528, 1997.

6. Bai S, Du L, Liu W, Whittle IR and He L: Tentative novel mechanism of the bystander effect in glioma gene therapy with HSV-TK/GCV system. Biochem Biophys Res Commun 259: 455-459, 1999.

7. Princen F, Robe P, Lechanteur C, Mesnil M, Rigo JM, Gielen J, Merville MP and Bours V: A cell type-specific and gap junctionindependent mechanism for the herpes simplex virus-1 thymidine kinase gene/ganciclovir-mediated bystander effect. Clin Cancer Res 5: 3639-3644, 1999.

8. Colombo BM, Benedetti S, Ottolenghi S, Mora M, Pollo B, Poli G and Finocchiaro G: The 'bystander effect': association of U-87 cell death with ganciclovir-mediated apoptosis of nearby cells and lack of effect in athymic mice. Hum Gene Ther 6: 763-772, 1995.

9. Mullen CA, Anderson L, Woods K, Nishino M and Petropoulos D: Ganciclovir chemoablation of herpes thymidine kinase suicide gene-modified tumors produces tumor necrosis and induces systemic immune responses. Human Gene Ther 9: 2019-2030, 1998.

10. Ram Z, Culver KW, Walbridge S, Blaese RM and Oldfield EH: In situ retroviral-mediated gene transfer for the treatment of brain tumor in rats. Cancer Res 53: 83-88, 1993.

11. Shinoura N, Chen L, Wani MA, Kim YG, Larson JJ, Warnick RE, Simon M, Menon AG, Bi WL and Stambrook PJ: Protein and messenger RNA expression of connexin43 in astrocytomas: implications in brain tumor gene therapy. J Neurosurg 84: 839-846, 1996. 
12. Wei SJ, Chao Y, Shih YL, Yang DM, Hung YM and Yang WK: Involvement of Fas (CD95/APO-1) and Fas ligand in apoptosis induced by ganciclovir treatment of tumor cells transduced with herpes simplex virus thymidine kinase. Gene Ther 6: 420-431, 1999.

13. Robe PA, Nguyen-Khac M, Jolois O, Rogister B, Merville MP and Bours V: Dexamethasone inhibits the HSV-tk/ganciclovir bystander effect in malignant glioma cells. BMC Cancer 5: 32, 2005.

14. Barkett M and Gilmore TD: Control of apoptosis by Rel/NFkappaB transcription factors. Oncogene 18: 6910-6924, 1999.

15. Zhang SQ, Kovalenko A, Cantarella G and Wallach D: Recruitment of the IKK signalosome to the p55 TNF receptor: RIP and A20 bind to NEMO (IKKgamma) upon receptor stimulation. Immunity 12: 301-311, 2000.

16. Robe PA, Bentires-Alj M, Bonif M, et al: In vitro and in vivo activity of the nuclear factor-kappaB inhibitor sulfasalazine in human glioblastomas. Clin Cancer Res 10: 5595-5603, 2004.

17. Otsuka G, Nagaya T, Saito K, Mizuno M, Yoshida J and Seo H: Inhibition of nuclear factor-kappaB activation confers sensitivity to tumor necrosis factor-alpha by impairment of cell cycle progression in human glioma cells. Cancer Res 59: 4446-4452, 1999.

18. Wahl C, Liptay S, Adler G and Schmid RM: Sulfasalazine: a potent and specific inhibitor of nuclear factor kappa B. J Clin Invest 101: 1163-1174, 1998.

19. Hermisson M and Weller M: NF-kappaB-independent actions of sulfasalazine dissociate the CD95L- and Apo2L/TRAIL-dependent death signaling pathways in human malignant glioma cells. Cell Death Differ 10: 1078-1089, 2003.

20. Robe PA, Princen F, Martin D, Malgrange B, Stevenaert A, Moonen G, Gielen J, Merville M and Bours V: Pharmacological modulation of the bystander effect in the herpes simplex virus thymidine kinase/ganciclovir gene therapy system: effects of dibutyryl adenosine 3',5'-cyclic monophosphate, alphaglycyrrhetinic acid, and cytosine arabinoside. Biochem Pharmacol 60: 241-249, 2000

21. Robe PA, Jolois O, N'Guyen M, Princen F, Malgrange B, Merville MP and Bours V: Modulation of the HSV-TK/ ganciclovir bystander effect by n-butyrate in glioblastoma: correlation with gap-junction intercellular communication. Int $\mathbf{J}$ Oncol 25: 187-192, 2004.

22. Grignet-Debrus C, Cool V, Baudson N, Velu T and CalbergBacq CM: The role of cellular- and prodrug-associated factors in the bystander effect induced by the Varicella zoster and Herpes simplex viral thymidine kinases in suicide gene therapy. Cancer Gene Ther 7: 1456-1468, 2000.

23. Malgrange B, Rigo JM, Coucke P, Belachew S, Rogister B and Moonen G: Beta-carbolines induce apoptotic death of cerebellar granule neurones in culture. Neuroreport 7: 3041-3045, 1996.
24. Delidow BC LJ, Peluso JJ and White B: Polymerase chain reaction. Vol. 58. Humana Press, Totowa, NJ, 1996.

25. Yamagishi N, Miyakoshi J and Takebe H: Enhanced radiosensitivity by inhibition of nuclear factor kappa B activation in human malignant glioma cells. Int J Radiat Biol 72: 157-162, 1997.

26. Piret B, Schoonbroodt S and Piette J: The ATM protein is required for sustained activation of NF-kappaB following DNA damage. Oncogene 18: 2261-2271, 1999.

27. Miyakoshi J and Yagi K: Inhibition of I kappaB-alpha phosphorylation at serine and tyrosine acts independently on sensitization to DNA damaging agents in human glioma cells. $\mathrm{Br} \mathrm{J}$ Cancer 82: $28-33,2000$

28. Weaver KD, Yeyeodu S, Cusack JC Jr, Baldwin AS Jr and Ewend MG: Potentiation of chemotherapeutic agents following antagonism of nuclear factor kappa B in human gliomas. J Neurooncol 61: 187-196, 2003.

29. Moriuchi S, Glorioso JC, Maruno M, Izumoto S, Wolfe D, Huang S, Cohen JB and Yoshimine T: Combination gene therapy for glioblastoma involving herpes simplex virus vector-mediated codelivery of mutant IkappaBalpha and HSV thymidine kinase. Cancer Gene Ther 12: 487-496, 2005.

30. Moriuchi S, Oligino T, Krisky D, Marconi P, Fink D, Cohen J and Glorioso JC: Enhanced tumor cell killing in the presence of ganciclovir by herpes simplex virus type 1 vector-directed coexpression of human tumor necrosis factor-alpha and herpes simplex virus thymidine kinase. Cancer Res 58: 5731-5737, 1998.

31. Hall SJ, Canfield SE, Yan Y, Hassen W, Selleck WA and Chen SH: A novel bystander effect involving tumor cell-derived Fas and FasL interactions following Ad.HSV-tk and Ad.mIL-12 gene therapies in experimental prostate cancer. Gene Ther 9: 511-517, 2002.

32. Albrecht H, Schook LB and Jongeneel CV: Nuclear migration of NF-kappa B correlates with TNF-alpha mRNA accumulation. J Inflamm 45: 64-71, 1995.

33. Kelly PJ, Daumas-Duport C, Kispert DB, Kall BA, Scheithauer BW and Illig JJ: Imaging-based stereotaxic serial biopsies in untreated intracranial glial neoplasms. J Neurosurg 66: 865-874, 1987.

34. Pallud J, Devaux B, Nataf F, Roux FX and Daumas-Duport C: Spatial delimitation of low grade oligodendrogliomas. Neurochirurgie 51: 254-259, 2005.

35. Robe PA, Martin D, Albert A, Deprez M, Chariot A and Bours V: A phase 1-2, prospective, double blind, randomized study of the safety and efficacy of Sulfasalazine for the treatment of progressing malignant gliomas: study protocol of [ISRCTN45828668]. BMC Cancer 6: 29, 2006. 